Pneumologe 2013 - 10 [Suppl 1]:7-9

DOI 10.1007/s10405-012-0657-0

Online publiziert: 19. Juni 2013

(c) Springer-Verlag Berlin Heidelberg 2013

\author{
N. Konietzko' $\cdot$ R. Loddenkemper ${ }^{2}$ \\ ${ }^{1}$ Essen \\ ${ }^{2}$ Berlin
}

\title{
10 Jahre Der Pneumologe
}

\section{Fortschritte in den letzten 10 Jahren und Perspektiven für die weitere Entwicklung in der Pneumologie}

\section{Habent sua fata libelli.}

Nicht nur Bücher, auch Zeitschriften haben ihre Geschichte.

Als vor 10 Jahren, im September 2004, das erste Heft dieser Zeitschrift mit dem Thema „Asthma“ gedruckt wurde, geschah dies in bewegten Zeiten. Kurz zuvor war die zertifizierte Weiterbildung für Ärzte obligat geworden und aller Orte sprießten CME-Aktivitäten wie Pilze nach einem warmen Regen aus dem Boden. So war auch das erklärte Ziel der neuen Zeitschrift zu verstehen, es lag ausschließlich in der Fort- und Weiterbildung. Im Konzept waren Themenhefte in 2-monatigem Rhythmus mit angeforderten Beiträgen vorgesehen. Die Abgrenzung zur "Pneumologie" als dem wissenschaftlichen Organ unseres Fachs erschien damit dem Verlag und den Begründern von Der Pneumologe eindeutig. Eine vergleichbare, gut funktionierende Arbeitsteilung gab es bereits in den meisten anderen medizinischen Fachgebieten.

Im Vorstand der Deutschen Gesellschaft für Pneumologie (DGP) freilich sah man das anders. Dort bestanden erhebliche Bedenken gegen eine weitere „Lungenzeitschrift". Man fürchtete eine Ausdünnung der wissenschaftlichen Substanz. In einem Schreiben vom Mai 2004 informierte der Geschäftsführer der DGP die Mitglieder des wissenschaftlichen Beirats von der Absicht des Springer-Verlags, im Herbst 2004 eine neue Zeitschrift herauszubringen, und ersuchte sie namens des Vorstands, von einer Mitarbeit abzusehen.

Das war natürlich „starker Tobak“. Letztlich war es ja die Initiative des DGPVorstands selbst gewesen, welche den Verlag auf die Idee zur Gründung einer neuen Zeitschrift gebracht hatte. Der Vorstand hatte beim Springer-Verlag angefragt, $\mathrm{ob}$ und unter welchen Konditionen eine pneumologische Zeitschrift als Organ der DGP realisiert werden könnte. Dies führte nach reiflichen Überlegungen zu einem detaillierten Angebot des Verlags an den DGP-Vorstand. Als dieses unbeantwortet blieb, wurde beschlossen, eine eigene pneumologische Zeitschrift herauszugeben.

Wir, die Mitbegründer der neuen Zeitschrift, sahen keinen Anlass zur Verzagtheit angesichts unserer prosperierenden Gesellschaft mit damals bereits mehr als 2000 Mitgliedern und einer breit fundierten wissenschaftlichen Expertise. Das Erscheinen einer neuen "Lungenzeitschrift" in einem seriösen Verlag wie Springer erschien uns vielmehr als Beweis der Attraktivität unseres Fachs. Auch erachteten wir es als bedeutsam, dass mit dem Bezug der Zeitschrift Der Pneumologe der Zugang zum damals größten CME-Portal im Internet verbunden war - und damit der Einstieg in das „E-Learning“.

In der Zwischenzeit hat sich der Pulverdampf längst verflüchtigt. Nach nunmehr 10 Jahrgängen mit jeweils 6 Heften lässt sich mit Fug und Recht sagen, dass sich die anfänglichen Befürchtun- 
gen nicht bewahrheitet haben. Das neue Journal wird viel und gern gelesen, auch von Nicht-Pneumologen, und es hat die pneumologische Landschaft im deutschsprachigen Raum bunter gemacht. Am Konzept hat sich seit der Gründung nichts verändert, allenfalls das äußere Erscheinungsbild und die stärkere Strukturierung mit neuen Rubriken. Nach dem Motto „Konkurrenz belebt das Geschäft" ist andererseits die Attraktivität der Pneumologie, des wissenschaftlichen Organs der DGP, sogar gestiegen.

So können Herausgeber und Verlag nach 10 bewegten Jahren getrost eine positive Bilanz ziehen. Nach vielerlei Überlegungen, wie man ein Jubiläum wie dieses am besten begehen sollte, erschien uns der akademische Weg der Geeignetste. Wir wollen mit diesem Heft eine wissenschaftliche Bilanz der letzten Dekade ziehen und Fortschritte bei wichtigen pneumologischen Krankheitsbildern und einigen Spezialgebieten beschreiben. Darüber hinaus sollen auch zukünftige Entwicklungen und die Potentiale unseres Fachs aufgezeigt werden. Für diese herkulische Aufgabe wurde die geballte Kraft von wissenschaftlichem Beirat und Herausgebern in die Pflicht genommen. Wir hoffen, dass wir mit den vorliegenden Beiträgen diesem Anspruch gerecht geworden sind. Den Autoren gehört großer Dank für die schnelle und sorgfältige Bearbeitung der Themen!

Bei den bedeutsamen Krankheiten unseres Fachs beginnt der Reigen mit der Volkskrankheit COPD. Immerhin 10\% aller Deutschen über 40 Jahre sind davon betroffen, vorwiegend Raucher, wie Greulich, Worth und Vogelmeier zeigen. Diagnostisch gewinnt neben dem Nachweis einer Atemwegsobstruktion die Computertomographie der Lunge an Bedeutung. Therapeutisch konnte mit der Einführung eines selektiven Phosphodiesterase-4-Inhibitors und von lang wirksamen Bronchodilatatoren ein kleiner, für zukünftige Entwicklung von Pharmazeutika aber möglicherweise wichtiger Schritt voran getan werden. In der Prävention des $\mathrm{Zi}$ garettenrauchens gab es zwar Fortschritte, jedoch sind weitere Verbesserungen notwendig.

Eine weitere Volkskrankheit unseres Fachs ist das Asthma bronchiale, wie Vir- chow ausführt. Unter den respiratorischen Erkrankungen blieb das Asthma auch in den letzten 10 Jahren, insbesondere bei Kindern, Jugendlichen und jungen Erwachsenen die häufigste chronische Erkrankung. Die Forschung zur Asthmatherapie umfasst vielversprechende Entwicklungen. Bessere Klassifizierung, gepaart mit zunehmend besserem Verständnis der zugrundeliegenden Mechanismen, darauf aufbauende, individualisierte Therapien und deren umfassende klinische Prüfung auf Praxistauglichkeit lassen hoffen, dass sich Morbidität und Mortalität des Asthma weiter senken lassen und die Erkrankten ein weitgehend normales Leben führen können.

\section{1) Asthma ist noch immer die häufigste chronische Erkrankung bei Kindern, Jugendlichen und jungen Erwachsenen}

Der Lungenkrebs, vorwiegend Folge des Tabakkonsums, ist in Deutschland nach wie vor die häufigste Krebstodesursache, wie Ukena nachweist. Fortschritte konnten in der Früherkennung (Niedrigdosis-CT), in der pathologischen Charakterisierung der Tumoren und in der Diagnostik durch den Einsatz der Positronenemissionstomographie (PET) und des endobronchialen Ultraschalls (EBUS) erzielt werden. Bei potentiell kurablen Tumoren setzen sich multimodale Therapiekonzepte immer mehr durch, während beim metastasierten kleinzelligen Karzinom das Zeitalter der zielgerichteten Therapie eingeläutet wird.

Das vorwiegend asbestinduzierte maligne Pleuramesotheliom wird wegen seiner langen Latenzzeit noch für Jahrzehnte aktuell bleiben. Zum Erhalt der bestmöglichen Lebensqualität werden verschiedene Therapiemöglichkeiten kombiniert.

Bronchopulmonale Infektionen stellen weltweit und auch in Deutschland eines der größten Gesundheitsprobleme dar, das in seiner Bedeutung häufig unterschätzt wird, wie Welte und Lorenz ausführen. In ihrer Übersicht gehen sie auf den aktuellen diagnostischen und therapeutischen Standard bei den Infektionen der oberen und besonders der unteren Atemwege (Bronchitis, Bronchiolitis,
Bronchiektasenkrankheit sowie ambulant erworbene und nosokomiale Pneumonien) ein. Sie warnen dezidiert vor dem zunehmenden Einsatz von Antibiotika bei oft nur viral bedingten Infekten und betonen die Bedeutung der Grippeschutzimpfung und - bei Risikogruppen der Pneumokokkenimpfung. In den letzten Jahren ist es gelungen, durch eine bessere Risikostratifizierung und durch eine an dem zu erwartenden Erregerspektrum ausgerichtete Therapie die Behandlung bronchopulmonaler Infektionen zu verbessern.

\section{》) In westlichen Industrieländern nehmen die TB-Fälle seit Jahren ab}

Trotz erheblicher Fortschritte in den vergangenen 10 Jahren, besonders in der raschen Diagnostik der multiresistenten Tuberkulose (TB) mittels molekularer Techniken und in der Entwicklung neuer Antibiotika, ist die TB mit geschätzten 8,7 Mio. neuer Fälle und 1,4 Mio. Todesfällen 2011 nach wie vor eine weltweite Bedrohung, betonen Diel, Bauer und Schaberg. Im Gegensatz zur globalen Entwicklung sind in westlichen Industrieländern, wie in USA oder Deutschland, seit Jahren sinkende Fallzahlen zu beobachten. Einen deutlichen Fortschritt hat auch die Diagnostik der latenten TB-Infektion mit den Interferon-Gamma-ReleaseAssays (IGRAs) erzielt. An der Entwicklung einer effektiveren Vakzine arbeiten mehrere internationale Zentren, mit der Einführung ist aber erst in einigen Jahren zu rechnen.

Die interstitiellen Lungenkrankheiten umfassen ein breites Spektrum von über 150 akut oder chronisch verlaufenden Entitäten, die mit einer Entzündungsreaktion und/oder Fibrosierung des Lungenparenchyms einhergehen und die nach ätiologischen oder histopathologischen Kriterien differenziert werden. Nach Costabel ist in Zukunft durch die verbesserte Diagnostik mittels HR-CT und durch den demographischen Wandel mit einer Zunahme der Zahl an idiopathischer Lungenfibrose (IPF) erkrankten Patienten zu rechnen. Ein Schwerpunkt der klinischen Forschung in den nächsten Jahren wird 
besonders bei der IPF der Validierung von Serum-Biomarkern zur Diagnose, der Prognoseabschätzung und der Evaluation des therapeutischen Ansprechens gewidmet sein. Auf dem therapeutischen Sektor werden mit Spannung die Ergebnisse der derzeit laufenden Phase-III-Studien erwartet. Wichtig ist, dass in Zukunft Therapiestudien nicht nur bei IPF-Patienten, sondern auch bei Patienten mit anderen progressiven Lungenfibrosen durchgeführt werden. Es ist damit zu rechnen, dass zunehmend mehr Patienten im Endstadium einer Lungenfibrose eine Lungentransplantation erhalten werden.

In kaum einem Bereich der Medizin hat sich in den vergangenen 10 Jahren der Wissenszuwachs so rasant entwickelt wie bei der pulmonalen Hypertonie, in erster Linie dank der Entwicklung neuartiger Therapien. Olschewski zeigt in seinem Beitrag, dass diese innovativen Behandlungskonzepte über ihre großen Erfolge hinaus auch einen Forschungs- und Wissensschub in der Genetik, Epidemiologie und Diagnostik der pulmonalen Hypertonie nach sich gezogen haben und für die nahe Zukunft weitere positive Überraschungen bereithalten.

Die zystische Fibrose ist längst keine Kinderkrankheit mehr. In Deutschland ist gut die Hälfte der Patienten erwachsen, wie Eickmeier, Smaczny, Gappa, Hirche und Wagner zeigen. Nach wie vor wird die Prognose wesentlich vom Verlauf der pulmonalen Manifestation bestimmt, die Therapie einschließlich der Transplantation ist weitgehend unverändert geblieben. Während die Gentherapie die in sie gesetzten Erwartungen nicht erfüllt hat, sind vom Verständnis der genetischen Störung abgeleitete Substanzen für kleine Gruppen der CF-Patienten bereits verfügbar oder stehen unmittelbar vor der Zulassung.

Unter den wichtigen Spezialgebieten unseres Fachs zeigen Pfeifer, Meyer, und Schönhofer die schier unglaublichen Fortschritte in der pneumologischen Intensivmedizin auf, insbesondere bei der nichtinvasiven Beatmung. Stichwortartig seien genannt die protektive Beatmung, die Optimierung von PEEP und Sedierung, die Möglichkeiten der extrakorporalen Oxygenierung und der $\mathrm{CO}_{2}$-Elimination sowie die außerklinische und die palliative Beatmung.
In der pneumologischen Schlafmedizin konnte in der vergangenen Dekade, wie Teschler und Arzt nachweisen, die „Positive-Airway-Pressure“-Therapie“ optimiert werden. Neue Modalitäten wie Schrittmacher oder Stimulationsgeräte verschiedenster Art werden möglicherweise schon bald eine sinnvolle Ergänzung zur CPAP-Behandlung darstellen. Ein historischer Meilenstein für die Schlafmedizin ist die fächerübergreifende Akzeptanz schlafbezogener Atmungsstörungen im perioperativen Management und als Risikofaktor für Herz-Kreislaufkrankheiten.

Was sich in der interventionellen Pneumologie getan hat, schildert Herth anhand der technischen Fortschritte in der Bronchologie: Bei malignen Atemwegsstenosen thermische Verfahren und Stentimplantationen, beim Emphysem die endoskopische Lungenvolumenreduktion und beim Asthma die Radiofrequenzablation der glatten Muskulatur bei Asthmapatienten. In einem faszinierenden Szenario beschreibt er abschließend zukünftige Entwicklungen in Diagnostik und Therapie. Für die internistische Thorakoskopie/ Pleuroskopie wurden semirigide/flexible Instrumente eingeführt; Hauptindikationen sind heute die Diagnostik von Pleuraergüssen und die Talkumpleurodese maligner Ergüsse.

Die erfreuliche Entwicklung unseres immer noch jungen Journals wäre nicht möglich gewesen ohne die vertrauensvolle Zusammenarbeit von Verlag und Herausgebern. Hier sei in erster Linie Frau Dr. Wieland gedankt, die das Projekt angestoßen und mit Geschick und Weitblick weitergeführt hat. Das Alltagsgeschäft mit der Redaktion lief stets reibungslos, namentlich zu nennen sind Frau Andreas, Frau Braun, Frau Dr. Kind, Frau Dr. Koch, Frau Dr. Schneider und Frau Dr. Schumacher. Die Arbeit mit ihnen war ein nie getrübtes Vergnügen und wird es hoffentlich noch lange bleiben. $\mathrm{Zu}$ danken ist natürlich der wissenschaftlichen Seele eines solchen Unterfangens, den Autoren, und letztendlich Ihnen, den Lesern von Der Pneumologe. Bleiben Sie unserer Zeitschrift treu, zumindest weitere 10 Jahre!

Nikolaus Konietzko

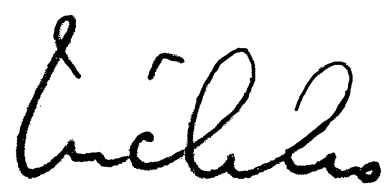

Robert Loddenkemper
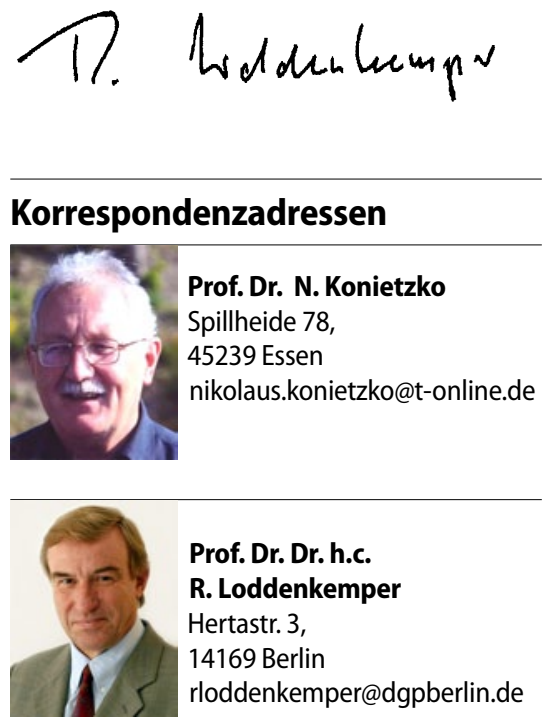